\title{
Concepção de um modelo matemático de avaliação de projetos de responsabilidade social empresarial (RSE)
}

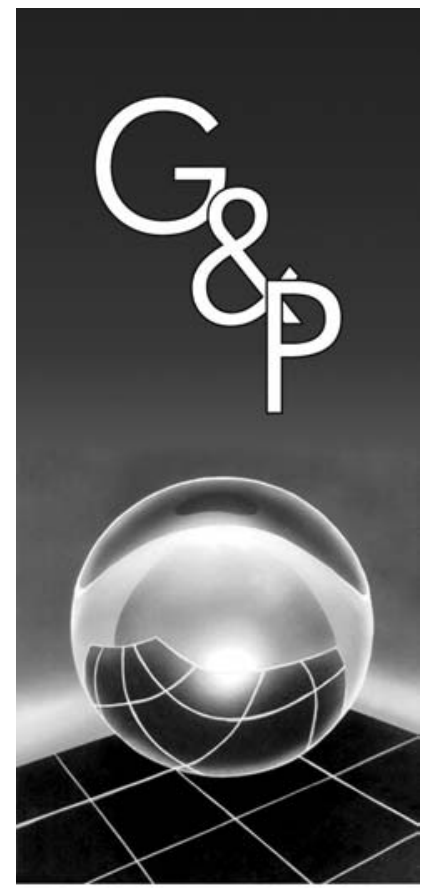

\author{
Katia Cristina Garcia \\ Marcello Goulart Teixeira \\ Cristiano da Costa Alves \\ Rex Nazaré Alves
}

\begin{abstract}
Resumo
O engajamento das empresas brasileiras em projetos de responsabilidade social empresarial (RSE) é cada vez maior, refletindo a consolidação de novas estratégias corporativas. Porém, a falta de modelos que permitam avaliar a adequação destes projetos cria um gargalo ao desenvolvimento e evolução da RSE. Isto porque estas iniciativas podem apresentar custos significativos que, não necessariamente, se traduzam em melhoria socioambiental para as comunidades afetadas pelas atividades da empresa. O presente trabalho propõe a avaliação de projetos de RSE por meio de um modelo matemático, construído a partir dos conceitos da lógica fuzzy (fuzzy logic), nebulosa ou lógica difusa. Desta forma, mostra-se que é possível integrar as diferentes dimensões do desenvolvimento sustentável na avaliação de projetos de RSE, gerando subsídios para tomada de decisão, ainda que os dados de entrada sejam incertos e de naturezas distintas.
\end{abstract}

Palavras-chave: Avaliação socioambiental. RSE. Modelagem matemática. Lógica fuzzy

\section{Introdução}

O conceito de desenvolvimento teve origem na Economia. Depois da Segunda Guerra Mundial, os economistas neoclássicos definiram o desenvolvimento como crescimento econômico e traçaram como objetivo principal a maximização do Produto Interno Bruto (PIB). Anos mais tarde, no início da década de 80, a crítica ecologista, surgida no contexto da crise do desenvolvimento, apontava para uma reflexão mais ampla. $\mathrm{O}$ enfoque ecologista mostrava que a utilização do modelo de crescimento econômico seguido pelos países industrializados não era possível em escala planetária, principalmente pela limitação dos recursos naturais. Este modelo também não era desejável, devido à degradação social e ambiental causada por seus modos de produção. O chamado "desenvolvimento maligno" deveria ser substituído pelo "ecodesenvolvimento", no qual o crescimento econômico seria buscado em conjunto com a preservação ambiental. Além das dimensões ecológica e econômica, o ecodesenvolvimento apresentava também dimensões sociais e culturais, sempre caminhando no sentido da autonomia, do desenvolvimento endógeno, self-reliant, e do planejamento participativo.

Em 1987, o Relatório "Nosso Futuro Comum", conhecido como o relatório de Brundtland, cunhou oficialmente o termo "desenvolvimento sustentável", prevendo a garantia de recursos naturais para as gerações futuras (COMISSÃO MUNDIAL SOBRE MEIO AMBIENTE E DESENVOLVIMENTO, 1988). A partir de então, planejar o desenvolvimento passou a significar a consideração simultânea das três dimensões da sustentabilidade: social, ambiental e econômica.

As críticas a este novo desenvolvimento estão focadas na falta de definição de quem seriam os sujeitos desta transformação. Certamente o Estado tem um papel fundamental na promoção deste desenvolvimento, mas não se pode deixar de atribuir uma importante parcela às empresas. Estas podem integrar, de maneira voluntária, as preocupações sociais e ambientais com suas atividades produtivas, em consonância com os princípios do desenvolvimento sustentável. Isto significa identificar os principais impactos sociais e ambientais causados por suas atividades, permitindo o estabelecimento de ações que visem a minimizá-los e mitigá-los, de forma a melhorar a qualidade de vida da população, considerando sua área de influência. Neste contexto é que estão inseridos os projetos de responsabilidade social empresarial (RSE). 
Diante da crise mundial de confiança nas empresas, que se instalou a partir dos anos 80 (MIFANO, 2002), a promoção de ações socioambientais que buscam uma melhoria da qualidade de vida dos trabalhadores e das comunidades do entorno vem sendo percebida de forma positiva pelo consumidor e pela sociedade, valorizando a marca da empresa (RICO, 2004; IPEA, 2001). Segundo Rico (2004), a conseqüência de um projeto de RSE bem sucedido é o seu reconhecimento institucional, comunitário e social. Porém, para que isto ocorra, é preciso que o projeto contemple ao longo de todo o seu ciclo de vida - desde a concepção até à execução e monitoramento as várias dimensões do desenvolvimento sustentável. Assim, é preciso sempre avaliar a adequação das ações em relação aos princípios da sustentabilidade social, ambiental e econômica (Quadro 1).

Além disso, a avaliação dos projetos de RSE mostra-se essencial para melhorar o desempenho durante a execução, e também no planejamento de projetos futuros, reduzindo as incertezas e riscos de insucesso. De acordo com Cohen e Franco (1998), a avaliação é uma etapa importante de qualquer projeto, pois tem como objetivo maximizar sua eficácia no alcance dos objetivos e também a eficiência na alocação de recursos para sua consecução.

Apesar da avaliação de projetos ser um processo bastante conhecido e usual, as metodologias e publicações sobre o tema apresentam uma abordagem restrita às questões econômicas, ainda com uma visão neoclássica do desenvolvimento, com foco para o estudo do mercado, o tamanho e localização do projeto, a engenharia, os custos e receitas e para a avaliação financeira e econômica (HOLANDA, 1969; BUARQUE, 1984; MAGALHÃES, 1986; FRASSON, 2001). Dentre estas metodologias, a Análise Custo-Benefício (ACB) é a mais conhecida e utilizada (BUARQUE, 1984; CONTADOR, 1988), mas também se podem mencionar o enfoque UNIDO (DASGUPTA et al., 1972), a avaliação social de projetos, também chamada de ASP, metodologia do banco

Quadro1. Princípios de sustentabilidade gerais

Sustentabilidade social - redução da desigualdade entre os padrões de vida, melhor distribuição da renda, atendimento às necessidades materiais e imateriais, busca de processos de produção que mantenham e respeitem as raízes e as particularidades de cada cultura e de cada local, controle, mitigação e compensação dos impactos sociais negativos.

Sustentabilidade ambiental - priorização do uso de recursos naturais renováveis, pesquisa, desenvolvimento e utilização de tecnologias menos poluidoras, conservação e reciclagem de recursos e energia, legislação efetiva de proteção ambiental, controle, mitigação e compensação dos impactos ambientais negativos, educação ambiental.

Sustentabilidade econômica - alocação e gestão de recursos de forma eficiente, macrossocial e não microempresarial, com maior fluxo de investimentos. mundial ou da OCDE (LITTLE; MIRRLEES, 1968; OCDE, 1975) e a metodologia de Harberger conhecida como "Escola de Chicago" (HARBERGER, 1973).

Devido à insipiência do processo de avaliação de projetos sociais e a necessidade em conhecer seus efeitos e resultados, empresas privadas, agências doadoras e financiadoras da cooperação internacional têm utilizado as metodologias acima citadas, para avaliar os projetos por elas apoiados. No entanto, vem sendo cada vez mais reconhecida a inadequação e limitação de tais metodologias na avaliação de projetos sociais (SILVA, 1997; COHEN; FRANCO, 1998; FRASSON, 2001). Neste sentido, Silva (1997) destaca que a avaliação com viés estritamente econômico mostra-se incapaz de dar conta da complexidade e multidimensionalidade do social, indicando a necessidade de novas metodologias que supram as lacunas evidenciadas pela aplicação de avaliações consideradas tradicionais.

Frasson (2001) busca uma direção para estas novas metodologias, e sugere que a tipologia mais adequada é aquela que permite uma avaliação dos projetos sociais tanto ex-ante quanto ex-post, devendo também ter a função de avaliar os impactos (avaliação somativa) ou processos (avaliação formativa), de forma mista ou participativa, no que diz respeito à procedência dos avaliadores.

Este tipo de avaliação apresenta elevada complexidade, pois envolve questões como diferenças sociais, culturais, ambientais, além de diferenças entre as pessoas e a forma de interação com o meio em que vivem. Tais questões podem ser traduzidas em sistemas socioambientais, caracterizados pelo alto grau de incerteza, o que dificulta a sua modelagem, demandando um sistema de apoio que represente as preferências e caminhos à participação dos atores sociais na justificação dos juízos de valor e na obtenção das soluções dos problemas observados no processo de avaliação (FERNANDES, 1996).

No caso particular de avaliações de projetos de RSE, a natureza fortemente não linear dos problemas, a incerteza e a subjetividade inerentes aos dados, a imprevisibilidade, normalmente negligenciadas em modelos convencionais, mas de extrema importância na correta representação dos problemas, passam a ser tratadas quando da utilização de ferramental matemático adequado, como a lógica fuzzy.

Apesar do uso de modelos matemáticos e computacionais ser ainda restrito nas ciências sociais, principalmente devido à pouca interação entre as áreas de conhecimento envolvidas, observa-se que a representação de sistemas e fenômenos do mundo real por meio de modelos matemáticos é possível, e tem mostrado grandes avanços nos últimos anos (PELLI NETO, 2004). Um exemplo é a lógica fuzzy desenvolvida por Zadeh na década de 1960. Tal Lógica tem sido utilizada de forma crescente na modelagem de problemas sociais devido a suas características peculiares, tais como: 
a) permitir a inclusão de incertezas não estocásticas no modelo;

b) facilitar o diálogo entre os especialistas do problema a ser tratado e o profissional de ciências exatas;

c) considerar de modo eficiente o conhecimento desses especialistas; e

d) modelar problemas complexos e não-lineares de modo simples.

Neste trabalho, é apresentado um modelo de avaliação dos projetos de RSE baseado na lógica fuzzy. Serão consideradas as dimensões social, ambiental e econômica da sustentabilidade, além da percepção dos diferentes atores sociais envolvidos.

A seção 2, a seguir, apresenta uma breve introdução à lógica fuzzy e a seção 3, o modelo desenvolvido pelos autores para avaliação de projetos de RSE. Na seção 4, são apresentados três exemplos de avaliação de projetos de RSE utilizando o modelo proposto por este trabalho. Por fim, na seção 5, encontram-se as conclusões e, na seção 6, as referências bibliográficas.

\section{Conceitos básicos de lógica fuzzy}

A lógica fuzzy, também conhecida como difusa, tem sua origem nos estudos feitos na década de 1960 por Lotfi A. Zadeh. O objetivo maior destes estudos foi modelar problemas de natureza industrial, biológica ou química, que envolvessem situações ambíguas e cujo processamento por meio da lógica computacional fundamentada na lógica booleana era inviável (ZADEH, 1965).

Em 1965, Zadeh publicou um artigo no qual avaliava o grau de pertinência de um determinado elemento em relação a um dado conjunto. Esta nova abordagem foi considerada inovadora, pois se diferenciava da lógica tradicional originária da filosofia grega, que verificava simplesmente o fato do elemento pertencer ou não ao conjunto. O grau de pertinência assume valores entre zero e um, inclusive, sendo que esses extremos representam, respectivamente, a completa exclusão e a total pertinência do elemento analisado em relação a um determinado conjunto fuzzy. É importante ressaltar que o grau de pertinência não representa uma medida ou valor probabilístico. Na verdade, é uma medida da compatibilidade do objeto analisado com o conceito representado pelo conjunto fuzzy (JOHN; REZA, 1999).

Deste modo, sendo $\mu_{\mathrm{A}}(\mathrm{x})$ a função de pertinência do elemento $\mathrm{x}$ no conjunto $\mathrm{A}$, cujo domínio é X e o contradomínio, o intervalo $[0 ; 1]$, um conjunto nebuloso A em $\mathrm{X}$ é um conjunto de pares ordenados, em que:

$$
\mathrm{A}=\left\{\left(\mathrm{x}, \mu_{\mathrm{A}}(\mathrm{x})\right) \mid \mathrm{x} \in \mathrm{X}\right\}
$$

Este conjunto também pode ser representado de forma contínua por meio da própria função de pertinência. A Figura 1 ilustra três conjuntos nebulosos, utilizados no

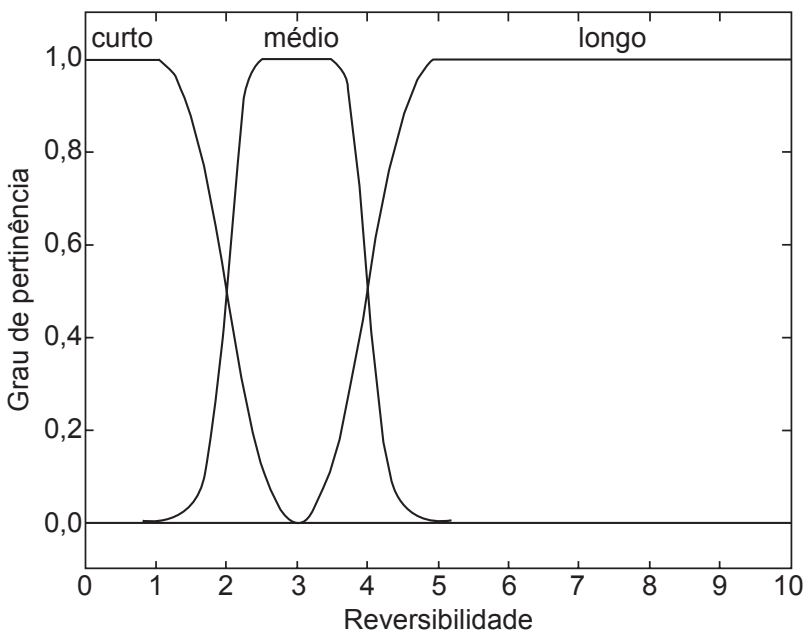

Figura 1. Exemplo de conjuntos nebulosos quanto à reversibilidade de projetos de RSE (curto, médio e longo prazo).

modelo matemático proposto neste trabalho, dados por curto, médio e longo, referentes à duração dos ganhos sociais obtidos com um projeto (reversibilidade). Assim, o eixo x representa a quantidade de anos e o eixo y o grau de pertinência desse valor em relação aos conjuntos acima citados. Nota-se, por exemplo, que uma duração entre 1 e 3 anos possui grau de pertinência diferente de zero em relação aos conjuntos curto prazo e médio prazo, e duração entre 3 e 5 em relação ao conjunto médio prazo e longo prazo.

A utilização de variáveis lingüísticas em substituição às variáveis numéricas representa outra grande inovação da lógica fuzzy em relação à tradicional lógica booleana. As variáveis lingüísticas admitem como valores expressões lingüísticas como "muito grande", "pouco frio", "mais ou menos jovem", que são representadas pelos conjuntos nebulosos.

Em essência, a utilização de descrição lingüística no lugar da descrição numérica permite o tratamento de fenômenos que são muito complexos para serem analisados por meio de termos matemáticos convencionais, permitindo modelar melhor os processos de tomada de decisão e avaliação (MOHAMED; CÔTÉ, 1999).

Ao associar-se um conjunto fuzzy a uma variável lingüística, restringe-se o valor de tal variável, assim como nos conjuntos booleanos. Porém, nos conjuntos nebulosos a noção de valores possíveis e impossíveis torna-se uma questão gradual. Apesar de serem similares em certos aspectos, existem diferenças conceituais importantes entre distribuições de possibilidade e de probabilidade. Enquanto a possibilidade está relacionada ao grau de facilidade com o qual uma variável assume um determinado valor, a probabilidade determina a chance de uma variável assumir o valor. Portanto, elas lidam com tipos diferentes de incerteza. A teoria da possibilidade trabalha fundamentalmente com a imprecisão, já a teoria 
da probabilidade, com as chances de ocorrência (JOHN; REZA, 1999; COX, 1994).

As relações entre variáveis lingüísticas de entrada e de saída são descritas por inferências fuzzy, mais comumente conhecidas como base de regras. Estas bases de regras utilizam proposições e conectivos lógicos tais como SE; ENTÃO; E; e OU. As inferências fuzzy fornecem conclusões ou saídas de um dado sistema analisando os níveis de compatibilidade das entradas com as condições impostas pela base de regras, ou seja, determinando como as regras serão ativadas e combinadas (OLIVEIRA Jr., 1999).

Para exemplificar uma inferência fuzzy, considere os conjuntos fuzzy $\mathrm{A}_{1}, \mathrm{~A}_{2}, \mathrm{~B}_{1}, \mathrm{~B}_{2}, \mathrm{C}_{1} \mathrm{e} \mathrm{C}_{2}$, as variáveis de entrada x e y e de saída $\mathrm{z}$. Uma possível base de regras seria

$$
\begin{aligned}
& \text { SE } \mathrm{x} \in \mathrm{A}_{1} \text { E } \mathrm{y} \in \mathrm{B}_{1} \text { ENTÃO } \mathrm{z} \in \mathrm{C}_{1} \\
& \text { SE } \mathrm{x} \in \mathrm{A}_{2} \text { E } \mathrm{y} \in \mathrm{B}_{2} \text { ENTÃO } \mathrm{z} \in \mathrm{C}_{2}
\end{aligned}
$$

sendo que $\mathrm{x} \in \mathrm{A}$ resulta no grau de pertinência do elemento x em relação ao conjunto fuzzy A e os operadores lógicos E e OU são definidos mais comumente, respectivamente, pelo mínimo e pelo máximo entre os graus de pertinência, ou seja,

conectivo e: $\mathrm{x} \in \mathrm{A} \wedge \mathrm{y} \in \mathrm{B}=\min \left\{\mu_{\mathrm{A}}(\mathrm{x}), \mu_{\mathrm{B}}(\mathrm{y})\right\}$

$$
\text { conectivo ou: } \mathrm{x} \in \mathrm{A} \vee \mathrm{y} \in \mathrm{B}=\max \left\{\mu_{\mathrm{A}}(\mathrm{x}), \mu_{\mathrm{B}}(\mathrm{y})\right\}
$$

Os resultados obtidos com a aplicação das diversas regras são agregados por meio do conectivo $\mathrm{OU}$, ou seja, são considerados os valores máximos para os graus de pertinência da variável de saída em relação a um determinado conjunto fuzzy.

A defuzzificação consiste na formalização de um dado número abrupto (não fuzzy) representativo do conjunto fuzzy resultante da aplicação da base de regras, sendo mais comumente utilizado o COG (center of gravity), que fornece o baricentro do gráfico do conjunto fuzzy obtido pela agregação, como pode ser observado na Figura 2.

\section{Sistema fuzzy para avaliação de projetos de RSE}

Neste trabalho, foi desenvolvido um sistema de avaliação de projetos de RSE representado como uma rede fuzzy de três camadas (Figura 3), englobando uma avaliação da sustentabilidade socioambiental (dada pelos benefícios sociais e ambientais), uma avaliação da sustentabilidade econômica e a percepção dos stakeholders (atores sociais). A saída deste sistema é uma nota normalizada em escala logarítmica, que representa a avaliação final global do projeto.

O diagrama da Figura 3 representa a rede, ou sistema fuzzy desenvolvido, constando de três camadas, com suas

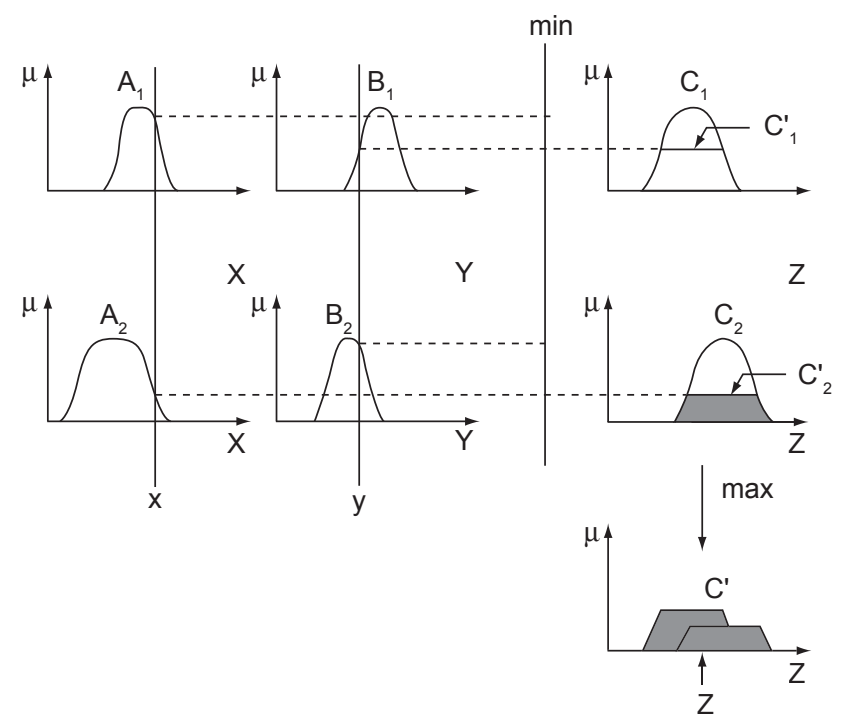

Figura 2. Exemplo de aplicação da base de regras e posterior defuzificação (JANG; SUN, 1995).

inferências fuzzy, $\mathrm{I}_{1}, \mathrm{I}_{3}$ e $\mathrm{I}_{2}, \mathrm{I}_{4}$ descritas nas Tabelas 1 e 2, respectivamente, e $\mathrm{I}_{5}$ na Tabela 3 . O objetivo do sistema é fornecer uma nota para os projetos de RSE avaliados, determinada a partir do benefício social e ambiental obtidos com a implantação dos projetos, dos custos e da percepção dos stakeholders, seguindo a metodologia proposta por Carvalho e Carvalho (2000). Os triângulos representam as inferências fuzzy $\mathrm{I}_{1}, \mathrm{I}_{2}, \mathrm{I}_{3}, \mathrm{I}_{4} \mathrm{e} \mathrm{I}_{5}$.

$\mathrm{O}$ sistema foi implementado computacionalmente utilizando o software MatLab. A descrição de cada etapa da avaliação é feita a seguir.

\subsection{Avaliação da sustentabilidade socioambiental}

Esta etapa pode ser realizada a partir da avaliação dos benefícios sociais e ambientais do projeto considerado, observando sua consonância com os princípios descritos no Quadro 1.

Segundo Carvalho e Carvalho (2000), os critérios para análise dos benefícios socioambientais podem ser estabelecidos de diversas formas. Neste trabalho, os benefícios sociais e ambientais são caracterizados por sua atratividade, abrangência e frequiência.

\subsubsection{Atratividade}

A variável atratividade, tanto social quanto ambiental, é resultado de uma inferência cujas variáveis de entrada são dadas pela reversibilidade do benefício e pelo ganho (CARVALHO; CARVALHO, 2000), podendo pertencer aos conjuntos fuzzy ruim, média e boa.

A primeira componente, a variável reversibilidade, representa o tempo ao longo do qual se espera obter os resultados desejados pelo projeto, e não necessariamente sua duração, e pode ser dado em anos ou em graus de 


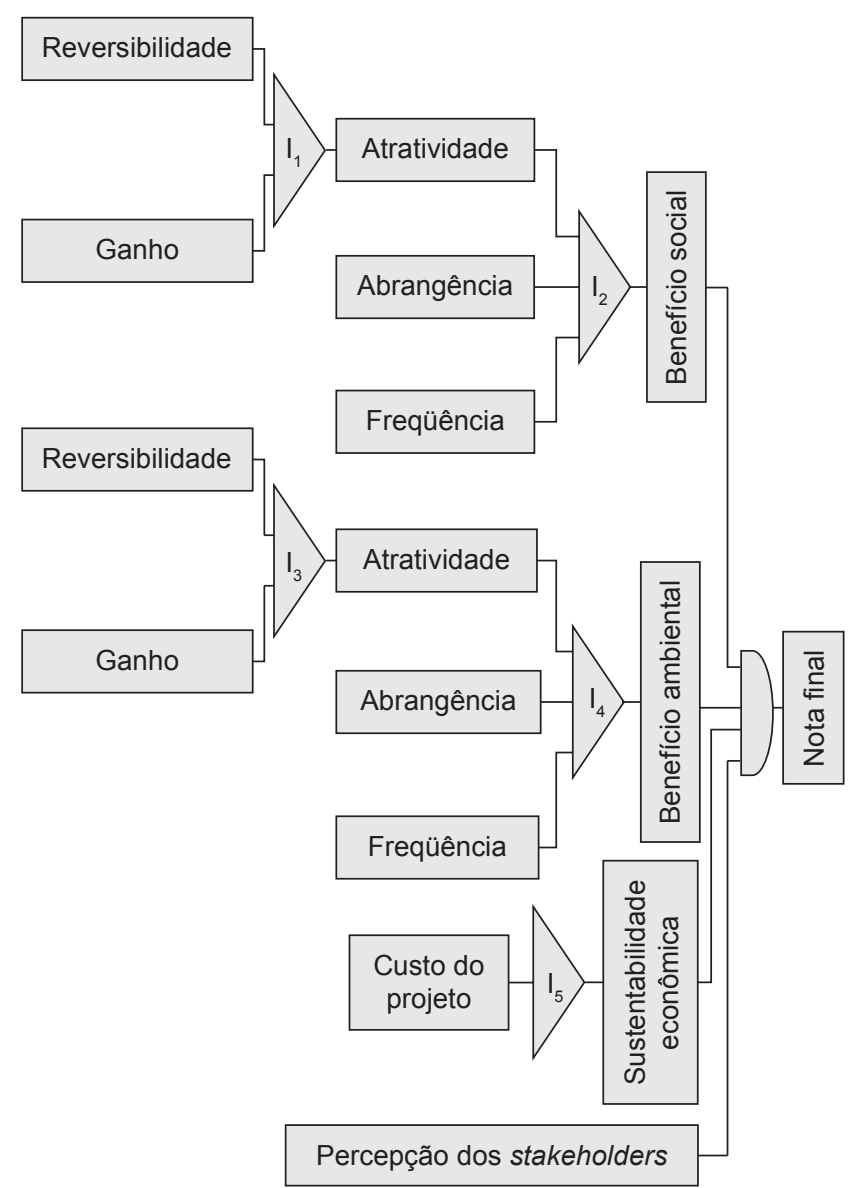

Figura 3. Sistema de avaliação social e ambiental de projetos de RSE.

Tabela 1. Base de regras para a variável atratividade.

\begin{tabular}{clc}
\hline SE reversibilidade $\in$ & OU ganho $\in$ & ENTÃO atratividade $\epsilon$ \\
\hline curto prazo & leve & baixa \\
médio prazo & moderado & média \\
longo prazo & expressivo & alta \\
\hline
\end{tabular}

pertinência em relação aos conjuntos curto prazo, médio prazo e longo prazo. Caso a entrada seja em anos, efetua-se uma fuzzyficação do valor da variável, ou seja, obtêm-se seus graus de pertinência em relação aos conjuntos fuzzy curto prazo, médio prazo e longo prazo por meio de funções pré-definidas por especialistas (Figura 1).

A segunda componente, a variável ganho, determina o nível de benefício social e ambiental esperado pelo projeto avaliado. Neste caso, são informados os graus de pertinência desta variável em relação aos conjuntos leves, moderados e expressivos.

A atratividade, juntamente com as variáveis abrangência e freqüência são, por sua vez, variáveis de entrada das inferências fuzzy $\mathrm{I}_{2} \mathrm{I}_{4}$, cujas saídas fornecem fatores, após a normalização, entre 1 e 10 para o benefício social e ambiental, respectivamente (Figura 3).
Tabela 2. Base de regras para os benefícios social e ambiental.

\begin{tabular}{|c|c|c|c|}
\hline $\begin{array}{c}\mathrm{SE} \\
\text { atratividade } \epsilon\end{array}$ & $\begin{array}{c}\mathbf{E} \\
\text { abrangência } \in\end{array}$ & $\begin{array}{c}\mathbf{E} \\
\text { frequiência } \in\end{array}$ & $\begin{array}{c}\text { ENTÃO } \\
\text { benefício } \epsilon\end{array}$ \\
\hline ruim & Isolada & esporádica & Trivial \\
\hline ruim & Isolada & ocasional & Moderado \\
\hline ruim & Isolada & freqüente & Substancial \\
\hline ruim & Limitada & esporádica & Trivial \\
\hline ruim & Limitada & ocasional & Moderado \\
\hline ruim & Limitada & freqüente & Substancial \\
\hline ruim & Ampla & esporádica & Moderado \\
\hline ruim & Ampla & ocasional & Substancial \\
\hline ruim & Ampla & freqüente & Substancial \\
\hline média & Isolada & esporádica & Moderado \\
\hline média & Isolada & ocasional & Substancial \\
\hline média & Isolada & freqüente & Importante \\
\hline média & Limitada & esporádica & Moderado \\
\hline média & Limitada & ocasional & Substancial \\
\hline média & Limitada & freqüente & Importante \\
\hline média & Ampla & esporádica & Moderado \\
\hline média & Ampla & ocasional & Substancial \\
\hline média & Ampla & freqüente & Importante \\
\hline boa & Isolada & esporádica & Substancial \\
\hline boa & Isolada & ocasional & Importante \\
\hline boa & Isolada & freqüente & Extremo \\
\hline boa & Limitada & esporádica & Substancial \\
\hline boa & Limitada & ocasional & Importante \\
\hline boa & Limitada & freqüente & Extremo \\
\hline boa & Ampla & esporádica & Substancial \\
\hline boa & Ampla & ocasional & Importante \\
\hline boa & Ampla & freqüente & Extremo \\
\hline
\end{tabular}

Tabela 3. Base de regras para a determinação da sustentabilidade econômica.

\begin{tabular}{lc}
\hline SE custo $\in$ & ENTÃO sustentabilidade econômica $\in$ \\
\hline baixo & pequena \\
médio-baixo & média-pequena \\
médio & média \\
médio-alto & média-alta \\
alto & alta \\
\hline
\end{tabular}

\subsubsection{Abrangência}

A variável abrangência representa o alcance geográfico do projeto e de seus benefícios, sendo informada por especialistas seus graus de pertinência em relação aos conjuntos fuzzy isolada, limitada e ampla. Uma vez que a variável está definida de forma genérica, o responsável pela análise deve considerar a região de atuação da empresa. Assim, para uma empresa que atue em nível municipal, por exemplo, um projeto de RSE será considerado com abrangência ampla quando este beneficiar todo o município ou extrapolar suas fronteiras. 


\subsubsection{Freqüência}

A variável frequiência determina com que periodicidade os ganhos sociais e ambientais são obtidos durante a vigência do projeto, se de forma esporádica, cíclica ou contínua ao longo do tempo (CARVALHO ; CARVALHO, 2000). Assim, na avaliação, são informados à rede fuzzy os graus de pertinência desta variável em relação aos conjuntos fuzzy esporádica, ocasional e freqüente.

Cabe enfatizar que as análises referentes ao benefício social e ambiental seguem a mesma lógica, mas são realizadas de forma independente. Um valor global será obtido posteriormente, a partir de uma composição das avaliações, conforme item 3.4.

Cabe lembrar que as funções de pertinência dos conjuntos fuzzy referentes às variáveis presentes na rede foram definidas por grupos de especialistas da área social e de meio ambiente. As bases de regras das duas inferências da rede foram construídas baseando-se na metodologia proposta por Carvalho e Carvalho (2000).

A Tabela 1 apresenta a base de regras das inferências $\mathrm{I}_{1}$ e I $\mathrm{I}_{3}$, cuja saída é a variável atratividade, constituída por três regras. São apresentados os conjuntos fuzzy, as variáveis linguísticas, em negrito, e os conectivos lógicos, em maiúsculas.

Ao ler a Tabela 1, pode-se identificar cada uma das três regras. Um exemplo de interpretação da primeira regra seria: se a reversibilidade, isto é, o tempo ao longo do qual se espera obter os ganhos do projeto, apresenta algum grau de pertinência no conjunto fuzzy curto prazo, ou o ganho social ou ambiental apresenta algum grau de pertinência no conjunto fuzzy leve, então a atratividade do projeto de RSE apresenta algum grau de pertinência no conjunto fuzzy baixa. Este grau de pertinência é determinado segundo a definição do conectivo OU, ou seja, o máximo entre os graus de pertinência das variáveis de entrada em relação aos conjuntos curto prazo e leve, respectivamente. A mesma lógica de interpretação pode ser aplicada às demais regras. Assim, após a aplicação dessas três regras obtêm-se os graus de pertinência da variável atratividade em relação aos conjuntos baixa, média e alta, que são, juntamente com as variáveis abrangência e frequiência, entrada das inferências $\mathrm{I}_{2}$ e $\mathrm{I}_{4}$.

As inferências $\mathrm{I}_{2}$ e $\mathrm{I}_{4}$, constituídas por 27 regras (Tabela 2), fornecem os graus de pertinência da variável benefício social e do benefício ambiental, respectivamente, em relação aos conjuntos trivial, moderado, substancial, importante e extremo (Figura 4). Depois da defuzzyficação pelo COG, os valores resultantes são normalizados no intervalo de 1 a 10, obtendo-se os fatores social $F_{S}$ e ambiental $F_{A}$, utilizados na determinação da nota final como descrito no item 3.4.

A Figura 5 ilustra a relação matemática entre as variáveis de entrada atratividade e abrangência e o fator do benefício social e ambiental (não normalizado no inter-

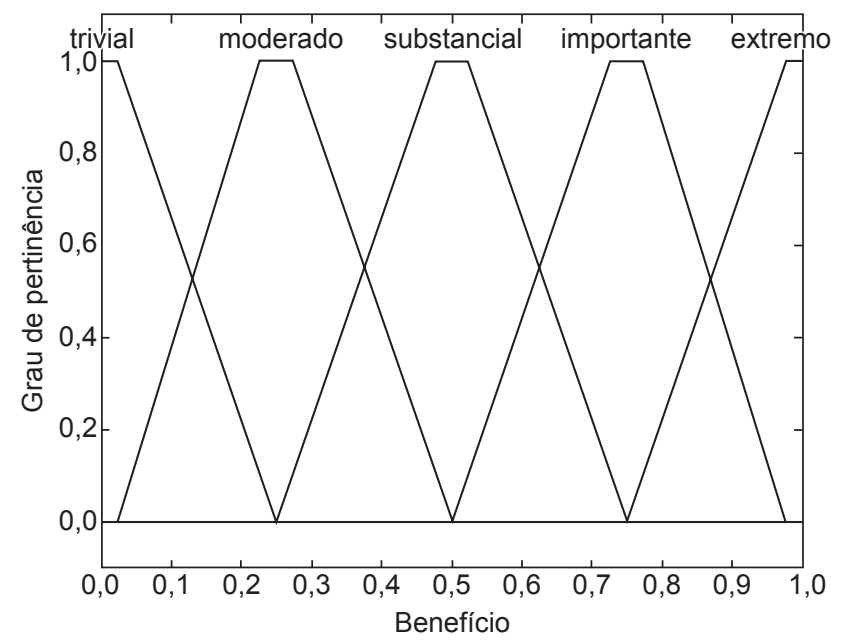

Figura 4. Funções de pertinências dos conjuntos fuzzy trivial, moderado, substancial, importante e extremo da variável benefício (social e ambiental).

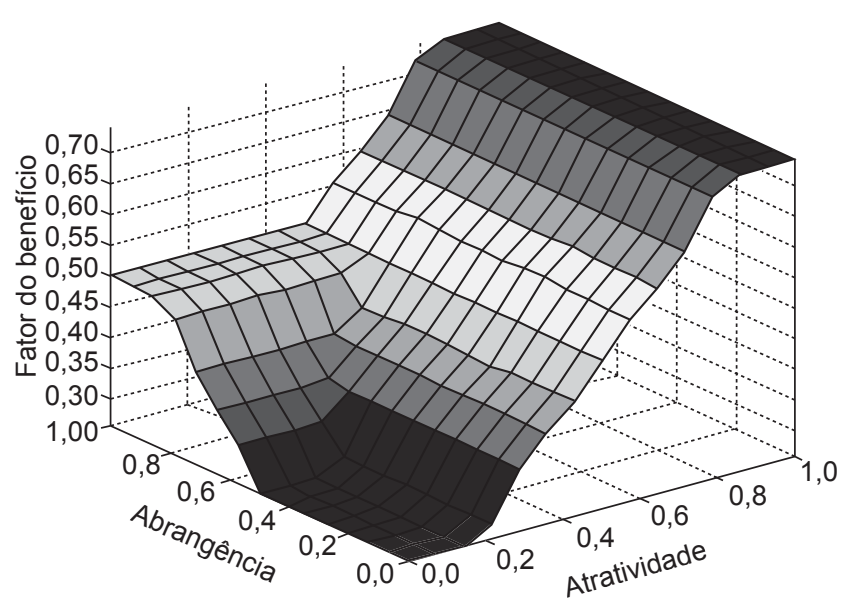

Figura 5. Relação entre as variáveis de entrada e de saída resultante da base de regras para o benefício social e ambiental.

valo de 1 a 10). Esta superfície é uma consequiência da base de regras apresentadas nas Tabelas 1 e 2, considerando-se a variável de entrada freqüência definida pelos graus de pertinência em relação aos conjuntos fuzzy esporádica, ocasional e freqüente iguais a $0,1 \mathrm{e}$ 0 , respectivamente. Nota-se que a forte não-linearidade desta relação e a impossibilidade de se obter a expressão da função que gera esta superfície por meio de médias ponderadas evidenciam a necessidade de utilização de uma metodologia baseada nos conceitos da lógica fuzzy, que permitem o tratamento de problemas de relação não linear entre as variáveis.

\subsection{Avaliação da sustentabilidade econômica}

Para a determinação da dimensão econômica, considera-se como variável de entrada o custo do projeto, em reais. De posse desse valor, obtém-se seu grau de perti- 
nência em relação aos conjuntos fuzzy baixo, médio-baixo, médio, médio-alto e alto, definidos de modo que a variável de entrada pertença a apenas um desses conjuntos. $\mathrm{O}$ grau de pertinência da variável de saída, relativo a um dos conjuntos fuzzy muito baixo, baixo, médio, alto e muito alto apresentados na Figura 6, é determinado segundo a base de regras apresentada na Tabela 3.

Por fim, o fator econômico $\mathrm{F}_{\mathrm{C}}$, utilizado na composição da nota final descrita na Seção 3.4, é obtido após a defuzzyficação pelo método COG da variável sustentabilidade econômica e posterior normalização no intervalo de 1 a 10 .

Ressalta-se que os conjuntos fuzzy das variáveis de entrada e de saída do cálculo da sustentabilidade econômica devem ser definidos por um grupo de especialistas da própria empresa, com base nos valores máximos e mínimos do histórico de projetos já implementados por ela. Assim, o modelo proposto torna-se viável para aplicação segundo a realidade financeira de empresas de pequeno, médio ou grande porte.

\subsection{Percepção dos diferentes stakeholders (atores sociais)}

A identificação da percepção dos stakeholders em relação ao projeto de RSE deve considerar um universo coerente com a variável abrangência, descrita anteriormente, variando de projeto para projeto. Uma nota deve ser estabelecida por cada representante dos grupos de interesse previamente identificados, a respeito da sua percepção sobre os benefícios socioambientais do projeto em questão. Isto pode ser feito por meio de questionário semi-estruturado, complementado com dados de entrevistas em campo. A partir de cada nota é estabelecida uma média. Uma vez obtida uma nota média, entre 1 e 10, que reflita a opinião da comunidade afetada, ou dos atores sociais relevantes, este valor é utilizado na fórmula

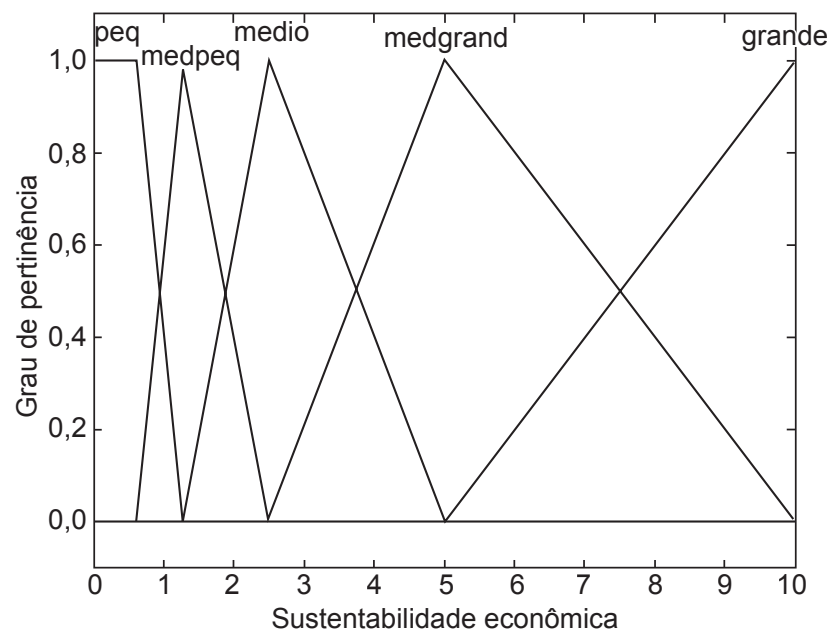

Figura 6. Relação entre as variáveis de entrada e de saída resultante da base de regras para a sustentabilidade econômica. de composição das avaliações, apresentada no próximo item.

\subsection{Composição das avaliações}

Os valores obtidos nas avaliações das dimensões social, ambiental e econômica, além do fator proveniente da identificação da percepção dos stakeholders, são utilizados para determinar uma nota, normalizada em escala logarítmica, que representa a avaliação final global do projeto de RSE. Este fator é proposto por:

$$
\mathrm{N}=\ln \left(\frac{\mathrm{p}_{\mathrm{S}} \mathrm{F}_{\mathrm{S}} \mathrm{p}_{\mathrm{A}} \mathrm{F}_{\mathrm{A}} \mathrm{p}_{\mathrm{O}} \mathrm{F}_{\mathrm{O}}}{\mathrm{p}_{\mathrm{C}} \mathrm{F}_{\mathrm{C}}}\right)
$$

em que $\mathrm{F}_{\mathrm{S}}$ e $\mathrm{F}_{\mathrm{A}}$ são os fatores social e ambiental, determinados respectivamente após a normalização no intervalo de 1 a 10 das saídas das inferências $I_{2}$ e $I_{4}$ (Figura 3); $\mathrm{F}_{\mathrm{O}}$ é o fator referente à opinião da comunidade, descrito em $3.3 ; \mathrm{F}_{\mathrm{C}}$, o fator de custo descrito em 3.2; e $\mathrm{p}_{\mathrm{S}}, \mathrm{p}_{\mathrm{A}}, \mathrm{p}_{\mathrm{O}}, \mathrm{p}_{\mathrm{C}}$, os pesos referentes a esses fatores respectivamente, considerados entre 1 e 10 e fornecidos por especialistas.

Normalizando o valor acima de modo que o valor máximo possível, ou seja, $\mathrm{F}_{\mathrm{S}}=\mathrm{F}_{\mathrm{O}}=\mathrm{F}_{\mathrm{A}}=10$ e $\mathrm{F}_{\mathrm{C}}=1$, seja igual a 10 e o valor médio, referente $\mathrm{a} \mathrm{F}_{\mathrm{S}}=\mathrm{F}_{\mathrm{O}}=\mathrm{F}_{\mathrm{A}}=\mathrm{F}_{\mathrm{C}}=5$, seja igual a 0 , independentemente dos valores dos pesos, chega-se à expressão:

$$
\begin{array}{r}
\overline{\mathrm{N}}=\frac{10\left(\mathrm{~N}-\mathrm{N}_{\text {médio }}\right)}{\mathrm{N}_{\text {máximo }}-\mathrm{N}_{\text {médio }}} \\
\text { em que } \mathrm{N}_{\text {médio }}=\ln \left(25 \frac{\mathrm{pp}_{\mathrm{O}} \mathrm{p}_{\mathrm{A}}}{\mathrm{p}_{\mathrm{C}}}\right) \\
\text { e } \mathrm{N}_{\text {médio }}=\ln \left(25 \frac{\mathrm{p}_{\mathrm{S}} \mathrm{p}_{\mathrm{O}} \mathrm{p}_{\mathrm{A}}}{\mathrm{p}_{\mathrm{C}}}\right)
\end{array}
$$

\section{Exemplos de aplicação da metodologia}

A metodologia desenvolvida neste trabalho foi aplicada a três projetos de RSE reais, com diferentes fatores sociais, ambientais, de custos e de percepção da comunidade, refletindo os diferentes graus de pertinência das variáveis em relação aos conjuntos fuzzy da rede.

Os dois primeiros referem-se a projetos sociais destinados a atender crianças, adolescentes e idosos de um município do Estado do Rio de Janeiro, e o terceiro, a um projeto de desenvolvimento sustentável que tem como objetivo gerar benefícios socioambientais decorrentes da construção de uma usina de geração de energia elétrica na região norte do país.

O fator de opinião da comunidade foi determinado a partir de entrevistas semi-estruturadas com os atores sociais beneficiados e com representantes da comunidade local e observações em campo. Os dados dos projetos são apresentados de forma resumida devido ao comprometi- 
mento da equipe com o sigilo das informações fornecidas pelas empresas. Cabe lembrar que o propósito deste trabalho não é realizar uma avaliação ex-post qualitativa, baseada na análise do cumprimento ou não das metas, objetivos e detalhamento dos projetos, mas sim propor e testar um modelo matemático, elaborado a partir de Carvalho e Carvalho (2000), que auxilie, de maneira esquemática e clara, o processo de avaliação de projetos de RSE. Desta forma, são apresentadas a seguir as informações essenciais para a validação do sistema de apoio à decisão proposto, relacionadas aos graus de pertinência das variáveis nos conjuntos fuzzy descritos anteriormente neste artigo. Tais informações são: linhas de ação do projeto (objetivo e público alvo); duração; custo total aproximado; e fator de opinião dos atores sociais relevantes (comunidade).

a) projeto 1: Atendimento à criança e ao adolescente;

- linha de ação: atenção à criança e ao adolescente de 2 a 17 anos e a suas famílias. Apoio médico e psicológico, palestras de educação sexual, oficinas de educação ambiental e reciclagem.

- duração: 1 ano. Custo total aproximado: $\mathrm{R} \$ 50.000,00$.

- fator de opinião da comunidade: 10,0.

b) projeto 2: Núcleo de convivência da terceira idade; e
- linha de ação: organização de centros comunitários para atividades culturais a pessoas da terceira idade. Desenvolvimento de projetos de arte com material natural (galhos, folhas, sementes). Criação e exposições de trabalhos para a comunidade.

- duração: 10 anos. Custo total aproximado: $\mathrm{R} \$ 30.000,00$

- fator de opinião da comunidade: 7,0.

c) projeto 3: Projeto de desenvolvimento sustentável.

- linha de ação: educação e qualificação da mão de obra local, fomento aos produtores rurais e melhoria da infra-estrutura urbana (infra-estrutura de saneamento básico).

- duração: 20 anos. Custo total aproximado: $\mathrm{R} \$ 1.000 .000,00$

- fator de opinião da comunidade: 7,0.

As Tabelas 4, 5 e 6 apresentam, para cada um dos três projetos, as médias dos graus de pertinência de cada variável em relação a seus conjuntos fuzzy, fornecidos por três especialistas. Os graus de pertinência das variáveis reversibilidade e custo foram determinados utilizando dados dos projetos. $\mathrm{O}$ fator $\mathrm{F}_{\mathrm{O}}$ foi obtido por meio de pesquisa de opinião junto à comunidade afetada pelo projeto. Consideram-se os pesos todos iguais a um.

Nota-se que o Projeto 2, apesar de um maior fator social $F_{S}$ e de um menor custo, obteve uma avaliação

Tabela 4. Graus de pertinência das variáveis Fuzzy, dos fatores e resultado final $\overline{\mathrm{N}}$ (valores numéricos truncados) para o Projeto 1.

\begin{tabular}{|c|c|c|c|c|c|c|c|c|c|}
\hline Projeto & Avaliação & Reversibilidade & Ganho & Freqüência & Abrangência & $F_{S}$ e $F_{A}$ & $\mathbf{F}_{0}$ & $\mathbf{F}_{\mathrm{C}}$ & $\overline{\mathbf{N}}$ \\
\hline \multirow{6}{*}{1} & Social & Curto prazo: 1,0 & Leves: 0,0 & Esporádica: 0,3 & Isolada: 1,0 & 6,6 & \multirow{6}{*}{10,0} & \multirow{6}{*}{1,8} & \multirow{6}{*}{6,0} \\
\hline & \multirow{5}{*}{ Ambiental } & Médio prazo: 0,0 & Moderados: 0,5 & Ocasional: 1,0 & Limitada: 0,2 & & & & \\
\hline & & Longo prazo: 0,0 & Expressivos: 1,0 & Freqüente: 0,7 & Ampla: 0,0 & \multirow{4}{*}{6,1} & & & \\
\hline & & Curto prazo: 0,0 & Leves: 0,0 & Esporádica: 0,3 & Isolada: 1,0 & & & & \\
\hline & & Médio prazo: 0,5 & Moderados: 0,8 & Ocasional: 1,0 & Limitada: 0,8 & & & & \\
\hline & & Longo prazo: 0,5 & Expressivos: 0,2 & Freqüente: 0,4 & Ampla: 0,0 & & & & \\
\hline
\end{tabular}

Tabela 5. Graus de pertinência das variáveis Fuzzy, dos fatores e resultado final $\overline{\mathrm{N}}$ (valores numéricos truncados) para o Projeto 2.

\begin{tabular}{|c|c|c|c|c|c|c|c|c|c|}
\hline Projeto & Avaliação & Reversibilidade & Ganho & Freqüiência & Abrangência & $F_{S}$ e $F_{A}$ & $\mathbf{F}_{0}$ & $\mathbf{F}_{\mathrm{C}}$ & $\overline{\mathbf{N}}$ \\
\hline \multirow{6}{*}{2} & Social & Curto prazo: 0,0 & Leves: 0,3 & Esporádica: 0,2 & Isolada: 0,6 & 7,5 & \multirow{6}{*}{7,0} & \multirow{6}{*}{1,5} & \multirow{6}{*}{4,8} \\
\hline & \multirow{5}{*}{ Ambiental } & Médio prazo: 0,0 & Moderados: 0,4 & Ocasional: 0,5 & Limitada: 1,0 & \multirow{5}{*}{4,3} & & & \\
\hline & & Longo prazo: 1,0 & Expressivos: 0,7 & Freqüente: 1,0 & Ampla: 0,2 & & & & \\
\hline & & Curto prazo: 1,0 & Leves: 0,8 & Esporádica: 0,3 & Isolada: 1,0 & & & & \\
\hline & & Médio prazo: 0,2 & Moderados: 0,1 & Ocasional: 1,0 & Limitada: 0,2 & & & & \\
\hline & & Longo prazo: 0,2 & Expressivos: 0,0 & Freqüente: 0,3 & Ampla: 0,0 & & & & \\
\hline
\end{tabular}

Tabela 6. Graus de pertinência das variáveis $F u z z y$, dos fatores e resultado final $\overline{\mathrm{N}}$ (valores numéricos truncados) para o Projeto 3.

\begin{tabular}{|c|c|c|c|c|c|c|c|c|c|}
\hline Projeto & Avaliação & Reversibilidade & Ganho & Freqüîncia & Abrangência & $\mathrm{F}_{\mathrm{S}}$ e $\mathrm{F}_{\mathrm{A}}$ & $F_{0}$ & $\mathbf{F}_{\mathrm{C}}$ & $\overline{\mathbf{N}}$ \\
\hline \multirow{6}{*}{3} & \multirow[t]{3}{*}{ Social } & Curto prazo: 0,0 & Leves: 0,0 & Esporádica: 0,0 & Isolada: 0,2 & \multirow[t]{3}{*}{8,1} & \multirow[t]{6}{*}{7,0} & 10,0 & \multirow[t]{6}{*}{1,3} \\
\hline & & Médio prazo: 0,0 & Moderados: 0,6 & Ocasional: 0,3 & Limitada: 0,5 & & & & \\
\hline & & Longo prazo: 1,0 & Expressivos: 0,8 & Freqüente: 0,8 & Ampla: 0,8 & & & & \\
\hline & \multirow[t]{3}{*}{ Ambiental } & Curto prazo: 0,3 & Leves: 0,0 & Esporádica: 0,0 & Isolada: 0,2 & \multirow[t]{3}{*}{7,2} & & & \\
\hline & & Médio prazo: 0,6 & Moderados: 0,5 & Ocasional: 0,2 & Limitada: 0,6 & & & & \\
\hline & & Longo prazo: 1,0 & Expressivos: 1,0 & Freqüente: 1,0 & Ampla: 1,0 & & & & \\
\hline
\end{tabular}


geral pior que o Projeto 1. Isto faz sentido uma vez que o fator ambiental foi bem menor, devido ao pequeno ganho obtido com iniciativas de educação ambiental para a terceira idade. Iniciativas de educação ambiental para crianças e adolescentes, como proposto no Projeto 1, tendem a trazer uma maior ganho, pois trabalham com horizontes de tempo mais amplos.

Observa-se também que apesar do Projeto 3 ter recebido as melhores avaliações no que diz respeito aos resultados sociais e ambientais, obteve a pior avaliação geral devido a seu alto custo. Para evitar este tipo de distorção, sugere-se efetuar comparações entre projetos que tenham uma mesma ordem de grandeza de custos, adaptando a definição dos conjuntos fuzzys muito baixo, baixo, médio, alto e muito alto para a variável custo, ou então fazer uma escolha apropriada para o peso $\mathrm{p}_{\mathrm{C}}$ do fator de custo $\mathrm{F}_{\mathrm{C}}$.

\section{Conclusão}

Neste trabalho, foi apresentado um modelo de avaliação de projetos de RSE utilizando os conceitos da lógica fuzzy. A utilização do modelo na avaliação de três projetos distintos possibilitou a manipulação de dados imprecisos, complexos e não-lineares. O modelo também permitiu incorporar no processo de avaliação o conhecimento de especialistas da área social, ambiental e da área de economia, além da percepção dos stakeholders. Apesar de representar uma quebra de paradigma em relação às metodologias de avaliação baseadas na lógica clássica, a teoria da lógica fuzzy facilita o diálogo entre o profissional de ciências exatas, responsável pela implementação computacional, e os especialistas das diversas áreas envolvidas, uma vez que permite a utilização de variáveis e expressões lingüísticas e de regras lógicas simples. Ressalta-se que a participação de um número maior de especialistas envolvidos na definição dos conjuntos fuzzy e na avaliação dos projetos resulta em respostas mais confiáveis.

Além disso, os dados de saída obtidos podem ser utilizados para subsidiar a tomada de decisão quanto aos investimentos em RSE. O modelo também pode ser utilizado para avaliar os benefícios socioambientais durante e no fim da implementação de um projeto, uma vez que a percepção dos atores sociais relevantes pode ser alterada ao longo de seu ciclo de vida.

\title{
Assessing corporate social responsibility projects through mathematical modeling
}

\begin{abstract}
The enrollment of Brazilian companies in Corporate Social Responsibility Projects (CSR) has increasead in the last years reflecting the consolidation of new corporative strategies. Nevertheless, the lack of models to assess these projects presents a problem to the development of CSR since the current projects can have significant financial costs which not necessarily bring social and environmental improvements to the communities directly affected by those companies. Thus, this paper proposes the assessment of CSR projects using a mathematical model based on fuzzy logic principles and the diffusion logic. Hence, it can be said that it is possible to integrate the different dimensions of the sustainable development when assessing CSR projects allowing decision-making even when the available input data are uncertain and distinct.
\end{abstract}

Keywords: Social and environmental assessment. CSR projects. Fuzzy logic.

\section{Referências bibliográficas}

BUARQUE, C. Avaliação Econômica de Projetos. 8. ed. Rio de Janeiro: Campus, 1984.

CARVALHO, A. B. M.; CARVALHO, M. A. B. A Determinação dos Impactos e a Gestão do Desempenho Social. Revista Meio Ambiente Industrial. São Paulo: Editora Tocalino, v. set/out. 2000.

COHEN, E.; FRANCO, R. Avaliação de projetos sociais. 2. ed. Petrópolis: Vozes, 1998.
COMISSÃO MUNDIAL SOBRE MEIO AMBIENTE E DESENVOLVIMENTO. Nosso Futuro Comum. Rio de Janeiro: Editora da Fundação Getúlio Vargas, 1988.

CONTADOR, C. R. Avaliação Social de Projetos. São Paulo: Ed. Atlas, 1988.

DASGUPTA, P. et al. Guidelines for Project Evaluation. New York, USA: Ed. UNIDO, 1972.

COX, E. The Fuzzy Systems Handbook. Estados Unidos: Academy Press Inc., 1994. 
FERNANDES, C. H. Priorização de Projetos Hidrelétricos sob a ótica social: um estudo de caso utilizando análise custo/ benefício e uma metodologia multicritério de apoio à decisão "MACBETH". Dissertação (Mestrado do Programa de Pós Graduação em Engenharia de Produção) - Universidade Federal de Santa Catarina, Florianópolis, SC, 1996.

FRASSON, I. Critérios de eficiência, eficácia e efetividade adotados pelos avaliadores de instituições não-governamentais financiadoras de projetos sociais. Dissertação (Mestrado do Programa de Pós Graduação em Engenharia de Produção) - Universidade Federal de Santa Catarina, Florianópolis, SC, 2001.

HARBERGER, A. C. (ed.). Project Evaluation: Collected Papers. Chicago, USA: Ed. Markham, 1973.

HOLANDA, N. Elaboração e avaliação de projetos. Rio de Janeiro, RJ: APEC, 1969.

IPEA. Pesquisa Ação Social das Empresas. Rio de Janeiro, RJ: IPEA, 2001.

JOHN Y.; REZA L. Fuzzy Logic: inteligence, control, and information. 1. ed. New Jersey, USA: Prentice Hall, 1999.

JANG, J.-R.; SUN, C.-T. Neuro-Fuzzy Modeling and Control. In: IEEE. S.1., Proceedings... v. 83, n. 3. 1995.

LITTLE, I. M.D.; MIRRLEES, J. A. Manual of Industrial Projects analysis for Developing Countries: Social costbenefit analysis, vol. II. Paris, FR: Ed. OCDE, 1968.

MAGALHÃES, F. C. Técnica de elaboração e avaliação de projetos. São Luís: UFMA; Fortaleza: BNB, 1986.
MIFANO, G. A crise mundial de confiança nas empresas: uma questão de Responsabilidade Social. (Evento da Bolsa de Valores de São Paulo, 14/08/2002). Disponível em: $<$ http://www.ethos.org.br/docs/conceito_praticas/publicações/ Debates/a_crisemundial.ppt>. Acesso em: ago. 2003.

MOHAMED, A. M. O.; CÔTÉ, K. Decision analysis of polluted sites - a Fuzzy set approach. Waste Management. United Kingdom: Pergamon, v. 19, n. 7, p. 519-533.1999.

OCDE. Manual de análise de projetos industriais: nos países em desenvolvimento, Estudo de casos. São Paulo, SP: Ed. Atlas, 1975.

OLIVEIRA JUNIOR, H. A. Lógica difusa: aspectos práticos e aplicações. Rio de Janeiro, RJ: Interciência, 1999.

PELLI NETO, A. Avaliação de imóveis urbanos com utilização de sistemas nebulosos (redes neuro-Fuzzy) e redes neurais artificiais. In: CONGRESSO PANAMERICANO DE VALUACIÓN, XXI., 2004. Anais... Cartagena, Colômbia, 2004.

RICO, E. M. A Responsabilidade Social Empresarial e o Estado: uma aliança para o desenvolvimento sustentável. São Paulo em Perspectiva. São Paulo, v. 18, n. 4, p. 73-82. 2004

SILVA, M. O. S. E. Avaliação de políticas sociais: concepção e modelos analíticos. Serviço Social \& Sociedade. São Paulo, v. ano 18, n. 53, p. 74-9, mar. 1997.

ZADEH, L. A. Fuzzy Sets. Information and Control. United Kingdom, v. 8, p. 338-353. 1965.

\section{Sobre os autores}

\section{Katia Cristina Garcia}

Coordenação dos Programas de Pós-graduação de Engenharia - COPPE,

Laboratório Interdisciplinar de Meio Ambiente - LIMA, Centro de Tecnologia,

Bloco I2000, Sala I208, Cidade Universitária, Universidade Federal do Rio de Janeiro - UFRJ,

Rio de Janeiro, RJ, Brasil,

e-mail: garciak@lima.coppe.ufrj.br

\section{Marcello Goulart Teixeira}

Departamento de Pós Graduação e Pesquisa em Sistemas e Computação,

Instituto Militar de Engenharia, Praça General Tibúrcio, 80, Praia Vermelha, Rio de Janeiro, RJ, Brasil,

e-mail: marcellogt@ime.eb.br

\section{Rex Nazaré Alves}

Departamento de Pós Graduação e Pesquisa em Engenharia Nuclear, Instituto Militar de Engenharia,

Praça General Tibúrcio, 80, Praia Vermelha, Rio de Janeiro, RJ, Brasil,

e-mail: rexnazare@ime.eb.br

\section{Cristiano da Costa Alves}

Departamento de Matemática, Fundação Educacional de Duque de Caxias - FEUDUC,

Av.Presidente Kennedy, 9422, D.Caxias, RJ, Brasil, e-mail: fisica2003@yahoo.com.br 